\title{
O impacto da Síndrome de Burnout entre os profissionais de saúde no contexto da pandemia da Covid-19
}

The impact of Burnout Syndrome among health professionals in the context of the Covid-19 pandemic

El impacto del Síndrome de Burnout entre los profesionales de salud en el contexto de la pandemia del Covid-19

Gabriel Martins Borges ${ }^{1 *}$, Jéssica Moreira Maia ${ }^{1}$, Paloma Oscar Xavier ${ }^{1}$, Alecsandra Barbosa dos Reis Santos ${ }^{1}$, Clélia Cristina Mourão Barbosa ${ }^{1}$, Vanessa Franquini Nogueira ${ }^{1}$, Alecxandra Mari Ito².

\section{RESUMO}

Objetivo: Identificar nas literaturas científicas o impacto da Síndrome de Burnout enfrentado pelos profissionais de saúde no contexto da pandemia da COVID-19. Métodos: Trata-se de uma pesquisa da literatura científica do tipo revisão integrativa. Para busca dos estudos foram utilizadas as bases de dados: Literatura Latino-americana e do Caribe em Ciências da Saúde (LILACS), National Library of Medicine (PUBMED), no período de 2020. Foram encontrados 124 estudos, sendo escolhidos para leitura 74 artigos, após a leitura e análise foram selecionados 10 artigos que relatavam os desafios e impactos acometidos pelos profissionais de saúde durante a pandemia. Resultados: Os estudos evidenciaram que os sentimentos vivenciados pelos profissionais de saúde que trabalham diretamente com pacientes infectados foram a ansiedade, o medo, estresse, exaustão, ausência de perspectivas relacionada a profissão, dificuldade nos relacionamentos laborais e familiares, visto que são fatores afetam nas dimensões da síndrome de Burnout. Considerações finais: Ao abordar sobre a Síndrome de Burnout no contexto da pandemia da COVID-19, os profissionais de saúde estão enfrentando níveis moderados e graves de Burnout e estão expostos a vários fatores agravantes biopsicossociais, devido as mudanças em seu cotidiano.

Palavras-chave: Burnout, Saúde mental, Coronavírus.

\begin{abstract}
Objective: To identify in the scientific literature the impact of the Burnout Syndrome faced by health professionals in the context of the COVID-19 pandemic. Methods: It is an integrative review research of scientific literature. To search for the studies, the databases were used: Latin American and Caribbean Literature in Health Sciences (LILACS), National Library of Medicine (PUBMED), in the period 2020. 124 studies were found, 74 articles were chosen for reading, after reading and analyzing 10 articles were selected that reported the challenges and impacts suffered by health professionals during the pandemic. Results: The studies showed that the feelings experienced by health professionals who work directly with infected patients were anxiety, fear, stress, exhaustion, lack of perspectives related to the profession, difficulty in work and family relationships, as the factors that affect the dimensions of Burnout syndrome. Final considerations: When approaching Burnout Syndrome in the context of the COVID-19 pandemic, health professionals are facing moderate and severe levels of Burnout and are exposed to several biopsychosocial aggravating factors, due to changes in their daily lives.
\end{abstract}

Key words: Burnout, Mental health, Coronavirus.

${ }^{1}$ Faculdade de Guarulhos (FAG), Guarulhos - SP.

2 Universidade Federal de São Paulo (UNIFESP), São Paulo - SP. * E-mail: gmborges44@gmail.com 


\section{RESUMEN}

Objetivo: Identificar en la literatura científica el impacto del Síndrome de Burnout que enfrentan los profesionales de salud en contexto de la pandemia de COVID-19. Métodos: Es una investigación de revisión integradora de la literatura científica. Para la búsqueda de los estudios se utilizaron las siguientes bases de datos: Literatura Latino-americana y del Caribe en Ciencias de la Salud (LILACS), Biblioteca Nacional de Medicina (PUBMED), en el período de 2020. Se encontraron 124 estudios, se seleccionaron 74 artículos para lectura, luego de leer y analizar 10 artículos fueron seleccionados, en él se informaron los desafíos e impactos que enfrentaron los profesionales de salud durante la pandemia. Resultados: Los estudios mostraron que los sentimientos vividos por los profesionales de salud que trabajan directamente con pacientes infectados fueron ansiedad, miedo, estrés, agotamiento, ausencia de perspectivas relacionadas con la profesión, dificultad en las relaciones laborales y familiares, ya que son factores que inciden en las dimensiones del Burnout síndrome. Conclusión: Al abordar el Síndrome de Burnout en el contexto de la pandemia COVID19, los profesionales de salud enfrentan niveles moderados y severos de Burnout y están expuestos a varios factores agravantes biopsicosociales, debido a cambios en su vida diaria.

Palabras clave: Burnout, Salud mental, Coronavirus.

\section{INTRODUÇÃO}

A Síndrome de Burnout (SB) é definida como uma condição de desconforto psicológico em resposta ao estresse de longa duração relacionado às condições desfavoráveis do ambiente de trabalho. Esta é caracterizada por esgotamento e exaustão psicológica, física, mental e que inclui três condições multidimensionais: exaustão emocional, despersonalização e baixa realização pessoal (GÜLER Y, et al., 2019).

O Burnout trata de um adoecimento produzido pelo contexto das estruturas e relações de trabalho, e tem sido mais frequentemente identificada em atividades relacionadas ao cuidar. Vem sendo bastante discutida nas literaturas científicas devido à complexidade de suas características e pela frequência de sua ocorrência na sociedade devido ao aumento das necessidades e demandas laborais na atual forma de organização do trabalho (GÜLER Y, et al., 2019).

A exaustão emocional é caracterizada pelo esgotamento físico e mental devido ao acúmulo de estresse que impacta em sua vida pessoal e profissional. O indivíduo passa a acreditar que não possui mais a energia para realizar suas tarefas habituais, levando-o a se sentir insatisfeito em seu local de trabalho e na sua realização pessoal. A despersonalização pode ser descrita como uma insensibilidade emocional, o indivíduo se desmotiva e começa a entender a vida como algo mecânico, e nessa dimensão, a ansiedade, irritabilidade e redução dos objetivos de autorrealização são manifestações comuns. A baixa realização pessoal é uma dimensão na qual acontece a autoavaliação negativa do indivíduo e afeta suas relações pessoas e também na realização das atividades laborais, criando conflitos entre o profissional, a equipe e até mesmo com os pacientes (RISSARDO MP e GASPARINO RC, 2013).

O quadro clínico é variado e pode apresentar sinais e sintomas mais gerais como fadiga, mialgia, distúrbios do sono, enxaqueca, quedas de cabelo. Entretanto, estudos mostram que há sintomas característicos que revelam impacto em sistemas do organismo específicos como trato gastrintestinal, cardiorrespiratório, neurológico e sexuais. Os sintomas psicológicos são destacados como falta de concentração, apatia, déficit de memória, baixa autoestima e agressividade. O quadro de Burnout implica também na maior probabilidade de absenteísmo e presenteísmo pelos profissionais acometidos (SILVEIRA ALP, et al., 2016).

A SB é um problema de saúde pública, mas vamos abordar mais especificamente seu impacto nos profissionais da área da saúde, uma vez que é um setor que presta serviços voltados a cuidados essenciais para a vida humana, principalmente neste momento em que o mundo enfrenta a pandemia pela SARS-CoV2, sendo estes profissionais identificados com alto risco de estresse ocupacional. Estes sintomas estão relacionados à natureza das atividades e à organização do trabalho. Distúrbios do sono, por exemplo, podem 
estar relacionados às longas jornadas de trabalho, baixo número de funcionários nas unidades, maior contato com pacientes críticos e seus familiares, por presenciarem momentos de dor e morte e pela carência de recursos que otimizam o processo de trabalho, o que consequentemente, possibilita o surgimento da Síndrome de Burnout (MESQUITA VSM e MALAGRIS LEN, 2020).

O novo coronavírus, denominado de SARS-CoV-2, um vírus de RNA, é o agente etiológico da COVID-19. O vírus foi detectado em dezembro de 2019 na China, em janeiro de 2020 a Organização Mundial da Saúde (OMS) declarou a epidemia uma emergência internacional, desencadeando bloqueios governamentais, distanciamento social, isolamento e outras medidas de interesse da saúde pública (LANA RM, et al., 2020).

De acordo com o Ministério da Saúde, globalmente houve aproximadamente 155 milhões de casos confirmados e mais de 3 milhões de mortes relatados. No Brasil, houve aproximadamente 15 milhões de casos e 410 mil óbitos por COVID-19, sendo 7.177,1/100mil habitantes e mortalidade de 199,4/100mil habitantes, visto que dentre as regiões do Brasil, o Sudeste tem a maior concentração de casos (BRASIL, 2021).

Os profissionais de saúde, durante a pandemia de COVID-19, se mostraram vulneráveis atuando na linha de frente da assistência aos pacientes acometidos com a doença. Existem fatores que contribuem como fontes de estresse e sofrimento psicológico relacionado ao momento atual da pandemia, sendo estes: a natureza da própria infecção, escassez de testes, número alto de admissões na Unidade de Terapia Intensiva (UTI), falta de um tratamento eficaz, evolução negativa de pacientes em estado grave, falta de Equipamento de Proteção Individual (EPI) e de suprimentos médicos (HORTA RL, et al., 2021).

Logo, de acordo com a importância e relevância do tema, este artigo teve como objetivo a construção de uma revisão integrativa da literatura, a fim de identificar e abordar o impacto da Síndrome de Burnout em profissionais de saúde, bem como os fatores contribuintes dentro do contexto do enfrentamento da pandemia da COVID-19.

\section{MÉTODOS}

Trata-se de uma revisão integrativa da literatura científica. Uma revisão integrativa visa a investigação de estudos originais, evidenciando os pontos relevantes dos estudos. É constituída por fases, sendo estas: a identificação da questão norteadora; o estabelecimento de critérios de inclusão e exclusão de estudos; a seleção, extração e categorização de informações obtidas dos artigos selecionados; a avaliação da qualidade, elegibilidade, análise e interpretação de resultados extraídos da pesquisa (SOUSA LMM, et al., 2018).

Para dar início ao estudo, foi formulada a seguinte questão norteadora: "O que a literatura tem apresentado acerca dos impactos da síndrome de Burnout entre os profissionais de saúde no contexto da pandemia da COVID-19?". Foram utilizadas bases de dados nacionais e internacionais, sendo elas: Centro LatinoAmericano e do Caribe de Informação em Ciências da Saúde (LILACS) e National Library of Medicine (PUBMED). A busca foi realizada através de palavras-chave cadastradas no portal de Descritores em Ciências da Saúde (DeCS), sendo eles: Burnout, Saúde Mental, Coronavírus. No cruzamento dos descritores, utilizou-se o operador booleano AND.

Os critérios de inclusão foram artigos publicados no ano de 2020 em português e inglês, disponíveis online e gratuitos na íntegra e que abordassem sobre o a síndrome de Burnout entre os profissionais de saúde no contexto da COVID-19. Foram excluídos do estudo: teses, dissertações, revisões, editoriais, artigos incompletos ou não disponíveis gratuitamente. A leitura, bem como a análise da qualidade metodológica dos estudos, foi realizada por cinco revisores desta pesquisa para garantir a avaliação crítica durante o processo de seleção dos artigos.

\section{RESULTADOS}

Através das buscas, foram encontrados 124 artigos, após a aplicação dos critérios de inclusão, foram excluídos 50 artigos com base no título e resumo, pois não correspondiam com o objetivo do estudo. Para a 
leitura foram analisados 74 artigos, na qual, após a leitura dos estudos, foram selecionados 10 artigos para subsidiar esta revisão (Figura 1).

Figura 1 - Fluxograma das etapas para a seleção dos artigos desta revisão integrativa, 2021.


Fonte: Borges GM, et al., 2021.

Quanto a seleção dos estudos, foram selecionados para esta revisão 10 artigos. A busca pelas bases de dados foi feita utilizando os filtros das próprias bases de dados para refinar a pesquisa dos artigos com base nos critérios de inclusão. As bases de dados que proporcionaram maior número de artigos relacionados ao objetivo foram: PUBMED (8 artigos, $80 \%$ ) e LILACS (2 artigos, 20\%).

Quanto ao tipo de estudo, identificou-se que 8 artigos (80\%) eram de estudos observacionais do tipo transversal, $1(10 \%)$ artigo de coorte prospectivo e $1(10 \%)$ artigo do tipo observacional descritivo. Quanto a origem dos estudos, apenas 1 artigo foi realizado no Brasil, 3 artigos na Europa e 6 artigos foram publicados na Ásia. Os artigos foram identificados e enumerados em ficha bibliográfica de acordo com os autores, periódico, tipo de estudo e objetivo (Quadro 1). 
Quadro 1 - Síntese de artigos selecionados quanto aos autores, periódico, tipo de estudo e objetivos, 2021.

\begin{tabular}{|c|c|c|c|c|}
\hline Id & Autores & Periódico & Tipo de Estudo & Objetivo \\
\hline 1 & LIU X, et al., 2020. & Frontiers in Psychiatry & Transversal & $\begin{array}{l}\text { Descobrir os fatores de associação, especialmente aqueles } \\
\text { intimamente relacionados a este surto, do desgaste do trabalho em } \\
\text { profissionais de saúde chineses. }\end{array}$ \\
\hline 2 & $\begin{array}{l}\text { RUIZ-FERNÁNDEZ MD, } \\
\quad \text { et al., } 2020 .\end{array}$ & $\begin{array}{l}\text { Journal of Clinical } \\
\text { Nursing }\end{array}$ & Transversal & $\begin{array}{l}\text { Avaliar a fadiga, o Burnout, a satisfação e o estresse percebido em } \\
\text { profissionais de saúde durante a crise de saúde da doença (COVID-19) } \\
\text { na Espanha. }\end{array}$ \\
\hline 3 & TAHERE SH, et al., 2020. & $\begin{array}{l}\text { Investigación y } \\
\text { educación en } \\
\text { enfermería }\end{array}$ & Transversal & $\begin{array}{l}\text { Avaliar o nível de Burnout no período de surto de COVID-19 e identificar } \\
\text { os fatores que influenciam entre enfermeiros da linha de frente e } \\
\text { enfermeiros de outras enfermarias. }\end{array}$ \\
\hline 4 & TAN BYQ, et al., 2020. & $\begin{array}{l}\text { Journal of the American } \\
\text { Medical Directors } \\
\text { Association }\end{array}$ & Transversal & $\begin{array}{l}\text { Examinar o Burnout e os fatores associados entre os profissionais de } \\
\text { saúde. }\end{array}$ \\
\hline 5 & MATSUO T, et al., 2020. & JAMA Network Open & Transversal & $\begin{array}{l}\text { Avaliar a prevalência de Burnout entre profissionais de saúde da linha } \\
\text { de frente durante a pandemia de COVID-19 no Japão com base nas } \\
\text { categorias de trabalho e outros fatores. }\end{array}$ \\
\hline 6 & GIUSTI EM, et al., 2020. & Frontiers in Psychology & Coorte prospectivo & $\begin{array}{l}\text { Identificar a prevalência de Burnout e sofrimento psíquico em } \\
\text { profissionais de saúde durante as fases iniciais da pandemia da COVID- } \\
\text { 19. }\end{array}$ \\
\hline 7 & $\begin{array}{l}\text { MARTÍNEZ-LÓPEZ JA, et } \\
\text { al., } 2020 .\end{array}$ & $\begin{array}{l}\text { Journal of Clinical } \\
\text { Medicine }\end{array}$ & $\begin{array}{l}\text { Descritivo e } \\
\text { quantitativo }\end{array}$ & $\begin{array}{l}\text { Verificar como a atual crise de saúde pela COVID-19 tem afetado os } \\
\text { profissionais de saúde, considerando as subescalas de Burnout de } \\
\text { Maslach e Jackson. }\end{array}$ \\
\hline 8 & $\begin{array}{l}\text { JOHNSON SU, et al., } \\
2020 .\end{array}$ & PLoS One & Transversal & $\begin{array}{l}\text { Avaliar a carga de saúde mental de profissionais de saúde que } \\
\text { trabalham direta e indiretamente com pessoas infectadas com o vírus } \\
\text { da COVID-19. }\end{array}$ \\
\hline 9 & HORTA RL, et al., 2020. & $\begin{array}{l}\text { Jornal Brasileiro de } \\
\quad \text { Psiquiatria }\end{array}$ & Transversal & $\begin{array}{l}\text { Investigar os efeitos da assistência na linha de frente da COVID-19 na } \\
\text { saúde mental de profissionais de hospital público. }\end{array}$ \\
\hline 10 & $\begin{array}{l}\text { ÇELMEÇE N e MENEKAY } \\
\text { M, } 2020 .\end{array}$ & Frontiers in Psychology & Transversal & $\begin{array}{l}\text { Determinar o efeito do estresse, ansiedade e níveis de Burnout de } \\
\text { profissionais de saúde (médicos, enfermeiros, auxiliares de saúde) que } \\
\text { cuidam de pacientes com COVID-19 na qualidade de vida. }\end{array}$ \\
\hline
\end{tabular}

Fonte: Borges GM, et al., 2021. 


\section{DISCUSSÃO}

Os artigos selecionados trouxeram vários fatores que implicam na saúde mental de profissionais de saúde que trabalham diretamente na linha de frente para o combate da pandemia da COVID-19. Esses profissionais têm em sua prática cotidiana várias situações de tensão que lhes exigem lidar frequentemente com 0 sofrimento, morte, escassez de recursos materiais para o cuidado, oscilações no quadro clínico de pacientes infectados, mudanças nos protocolos e a intensidade das demandas de pacientes admitidos. Com estas vivências, de maneira contínua, começam a surgir exacerbações acerca de esgotamento físico e psicológico (ARAGÃO NCS, et al., 2020).

Em um estudo transversal realizado no Irã por Tahere SH, et al. (2020), cujo objetivo foi identificar o nível de Burnout entre profissionais de saúde que estão trabalhando diretamente nas enfermarias para infecção por COVID-19 e profissionais de outras enfermarias habituais, demonstrou-se que o nível de esgotamento foi maior no grupo que estava lidando diretamente com os pacientes infectados, e como critério de maior impacto para o estresse no trabalho, associado a turnos mais longos, seguido pela escassez de recursos e estresse emocional pela distância de familiares e amigos. Em outro estudo, realizado por Barello S, et al. (2020) constatou-se que os níveis de exaustão emocional pareceram maiores do que os valores normativos e a porcentagem de trabalhadores com altos níveis de exaustão foi significativamente maior do que a encontrada em outras amostras antes do surto de COVID-19.

Quanto às três dimensões do Burnout, em um estudo transversal realizado na China em 2020, não houve diferença estatística entre as três dimensões em gênero e grupos ocupacionais. Entretanto, identificou-se que o local de trabalho (região de alta ou baixa admissão de pacientes com COVID-19) e o estilo de enfrentamento podem afetar a distribuição positiva das três condições multidimensionais do Burnout simultaneamente. Este estudo também evidencia que a taxa Burnout entre os profissionais foi de $73,98 \%$, aumento dos casos de grau moderado a severo durante a pandemia e que a distância dos profissionais de suas famílias devido ao isolamento social também pode ser um fator de esgotamento profissional (LIU X, et al., 2020).

Os trabalhadores de saúde mais jovens apresentam níveis mais elevados de estresse e ansiedade. Isso pode ser devido à falta de experiência de trabalho em situações estressantes semelhantes. Em um estudo transversal realizado na Itália, que teve como objetivo avaliar o nível de Burnout relacionado a fatores pessoais e ambientais em profissionais de saúde que trabalham em unidades de assistência a pacientes com COVID-19, identificou-se que os profissionais de saúde mais jovens (menos que 29 anos), solteiros ou com menos experiência prática são mais propensos à síndrome de Burnout. No geral, os profissionais de saúde em unidades que lidam diretamente com pacientes com Covid, por um período prolongado, experimentaram exaustão emocional moderada, alta despersonalização e sensação de realização pessoal altamente reduzida (WANG J, et al., 2020).

Uma escala bastante utilizada pelos estudos foi o Inventário de Burnout de Maslach (MBI). Esse instrumento possui 22 itens ou questões que analisam as três dimensões da Síndrome de Burnout, exaustão emocional, despersonalização e realização profissional (CAMPOS ICM, et al., 2020). No estudo de MartínezLópez JA, et al. (2020), utilizando-se essa escala, em uma amostra de 157 profissionais da saúde da UTI de diferentes hospitais espanhóis, cujo objetivo foi determinar o grau de Burnout e seus principais desencadeadores em profissionais de saúde na Espanha no período mais crítico da pandemia da COVID-19, os resultados apresentaram um número maior de profissionais acometidos com despersonalização através do questionário e que $43,3 \%$ dos entrevistados estimam que futuramente necessitarão de tratamento psicológico.

Em um estudo realizado na Noruega, do tipo transversal, em uma amostra de 178 médicos, 770 enfermeiras, 244 psicólogos clínicos e 78 outros profissionais de saúde, que trabalham diretamente com pacientes Covid-19, 36,5\% tinham sintomas clínicos ou subclínicos de Transtorno de Estresse PósTraumático (PTSD), em contraste com 27,3\% para aqueles que trabalhavam indiretamente e em outras unidades. A síndrome de Burnout foi positivamente associada aos sintomas de PTSD. O estudo também enfatiza a importância do monitoramento dos sintomas subclínicos entre os indivíduos que trabalham com pacientes com COVID-19 (JOHNSON SU, et al., 2020). 
No que tange a prevalência de Burnout em profissionais que possuíam autoridade de decisão diante de pacientes com níveis críticos de gravidade, nota-se que as decisões podem levar a dilemas éticos e morais, capazes de gerar sofrimento psicológico. Isso porque, tais decisões podem incluir o cuidado de pacientes em estado grave com limitação de recursos e ter que priorizar alguns casos em detrimento de outros. Outro fator do ambiente laboral que reflete em profissionais de saúde é a expetativa de apreciação pelos seus superiores. A importância do trabalho em equipe, com o uso de mensagens positivas e de suporte, tem sido de grande valia para a manutenção da saúde mental dos profissionais no ambiente de trabalho (MATSUO T, et al., 2020; RUIZ-FÉRNANDEZ MD, et al., 2020).

O apoio social é definido como interações ou relacionamentos sociais que fornecem aos indivíduos uma assistência ou que incorpora estes a um sistema social, visto que podem fornecer amor, cuidado ou senso de apego a um grupo. No estudo de Zhang $\mathrm{H}$, et al. (2020) destaca-se que o apoio social pode desempenhar um papel fundamental no alívio do efeito prejudicial do Burnout nos sintomas de ansiedade entre os profissionais de saúde. Isto porque, o meio social pode permitir que os indivíduos tenham um senso de previsibilidade e estabilidade em suas vidas, bem como o reconhecimento de sua autoestima, controle pessoal e domínio. Entretanto, no momento atual frente a pandemia, esse recurso social se encontra limitado, uma vez que uma das estratégias para controle da transmissão da COVID-19, é o isolamento social.

O estudo italiano de coorte prospectivo, mostrou que houve níveis muito elevados de estado de ansiedade, o que pode sugerir a presença de tensão, que pode iniciar ou agravar sintomas de esgotamento e sofrimento mental. Em relação ao Burnout, 107 (35,7\%) dos profissionais estudados apresentaram exaustão emocional moderada e 105 (31,9\%) severa e para a despersonalização temos que 46 (14,0\%) apresentaram níveis moderados e $40(12,1 \%)$ graves. Quanto ao aspecto de realização pessoal reduzida foram identificados $132(40,1 \%)$ profissionais com níveis moderados e $113(34,3 \%)$ graves. O mesmo estudo também abordou a influência positiva dos mecanismos institucionais de proteção para transmissão, tratamento do vírus e a redução da carga de trabalho (GIUSTI EM, et al., 2020).

Quanto ao perfil prevalente no que diz respeito aos sinais de estresse, traços de ansiedade e esgotamento mental, que são preditores para o desenvolvimento de Burnout, um estudo realizado com 240 profissionais de saúde atuando em hospitais na Turquia, obteve como resultado que a prevalência de tais sinais foi maior no sexo feminino em relação ao sexo masculino, além de observar que a presença de filhos foi um fator de grande relevância para o desenvolvimento dos mesmos (ÇELMEÇE N e MENEKAY M, 2020). Outros dois estudos, também evidenciaram a prevalência de sinais de exaustão emocional na população amostral do sexo feminino, relacionando-os também a profissão de enfermagem e a estar em contato direto com os pacientes (GIUSTI EM, et al., 2020; TAN BYQ, et al., 2020).

\section{CONSIDERAÇÕES FINAIS}

Ao abordar sobre a Síndrome de Burnout no contexto da pandemia da COVID-19, os estudos evidenciaram que os profissionais de saúde estão enfrentando níveis significativos de risco para Burnout por estarem expostos a severas mudanças em seu cotidiano. Isso pode gerar impactos psicológicos, resultando em exaustão emocional, despersonalização e baixa realização pessoal. Logo, essa pesquisa traz a relevância do estudo sobre Burnout durante a pandemia e a importância para que se realizem ações de intervenção na organização do trabalho. Também é interessante que se promovam mais estudos para investigar medidas de promoção à saúde mental, prevenção de Síndrome de Burnout e analisar as respostas a essas medidas no cotidiano do enfrentamento da pandemia da COVID-19.

\section{REFERÊNCIAS}

1. ARAGÃO NSC, et al. Burnout syndrome and associated factors in intensive care unit nurses. Revista Brasileira de Enfermagem, 2021; 74(3): e20190535.

2. BARELLO S, et al. Burnout and somatic symptoms among frontline healthcare professionals at the peak of the Italian COVID-19 pandemic. Psychiatry Research, 2020; 290: e113129. 
3. BRASIL. Ministério da Saúde. Painel Coronavírus, 2021. Disponível em: https://covid.saude.gov.br. Acesso em: 21 de maio de 2021.

4. CAMPOS ICM, et al. Maslach Burnout Inventory - Human Services Survey (MBI-HSS): revisão integrativa de sua utilização em pesquisas Brasileiras. Arquivos de Ciências da Saúde da UNIPAR, 2020; 24(3): 187-195.

5. ÇELMEÇE N, MENEKAY M. The effect of stress, anxiety and Burnout levels of healthcare professionals caring for COVID-19 patients on their quality of life. Frontiers in Psychology, 2020; 11: 597624.

6. GIUSTI EM, et al. The psychological impact of the COVID-19 outbreak on health professionals: a cross-sectional study. Frontiers Psychology, 2020; 11: e1684.

7. GÜLER Y, et al. Burnout syndrome should not be underestimated. Revista da Associação Médica Brasileira, 2019; 65 (11): 1356-1360.

8. HORTA RL, et al. O estresse e a saúde mental de profissionais da linha de frente da COVID-19 em hospital geral. Jornal Brasileiro de Psiquiatria, 2021; 70 (1): 30-38.

9. JOHNSON SU, et al. PTSD symptoms among health workers and public service providers during the COVID-19 outbreak. PLoS One, 2020; 15(10): e0241032.

10. LANA RM, et al. Emergência do novo coronavírus (SARS-CoV-2) e o papel de uma vigilância nacional em saúde oportuna e efetiva. Cadernos de Saúde Pública, 2020; 36 (3): e00019620.

11. LIU X, et al. COVID-19 Outbreak Can Change the Job Burnout in Health Care Professionals. Frontiers in Psychiatry, 2020; 11: 563781.

12. MARTÍNEZ-LÓPEZ JA, et al. Psychological Impact of COVID-19 Emergency on Health Professionals: Burnout Incidence at the Most Critical Period in Spain. Journal of Clinical Medicine, 2020; 9(9): e3029.

13. MATSUO T, et al. Prevalence of health care worker burnout during the coronavirus disease 2019 (covid-19) pandemic in Japan. JAMA Network Open, 2020; 3(8): e2017271.

14. MESQUITA VSM, MALAGRIS LEN. Síndrome de Burnout em residentes multiprofissionais em saúde de um hospital universitário. Revista de Sociedade Brasileira de Psicologia Hospitalar, 2020; 23(2): 65-76.

15. RISSARDO MP, GASPARINO RC. Exaustão emocional em enfermeiros de um hospital público. Escola Anna Nery, 2013; 17(1): 128-132.

16. SILVEIRA ALP, et al. Burnout Syndrome: consequences and implications of an increasingly prevalent reality in health professionals' lives. Revista Brasileira de Medicina do Trabalho, 2016; 14 (3): 275-284.

17. SOUSA LMM, et al. Revisões da literatura científica: tipos, métodos e aplicações em enfermagem. Revista Portuguesa de Enfermagem de Reabilitação, 2018; 1(1): 45-54.

18. TAHERE SH, et al. Burnout and its influencing factors between frontline nurses and nurses from other wards during the outbreak of Coronavirus Disease -COVID-19- in Iran. Investigación y Educación en enfermería, 2020 ; 38 (2): e03.

19. RUIZ-FERNÁNDEZ MD, et al. Compassion fatigue, burnout, compassion satisfaction and perceived stress in healthcare professionals during the COVID-19 health crisis in Spain. Journal of Clinical Nursing, 2020; 29: 4321-4330.

20. TAN BYQ, et al. Burnout and Associated Factors Among Health Care Workers in Singapore During the COVID-19 Pandemic. Journal of the American Medical Directors Association, 2020; 21(12): 1751-1758.

21. ZHANG H, et al. Anxiety symptoms and burnout among Chinese medical staff of intensive care unit: the moderating effect of social support. BMC Psychiatry, 2020; 20: 197.

22. WANG J, et al. Burnout syndrome in healthcare professionals who care for patients with prolonged disorders of consciousness: a cross-sectional survey. BMC Health Services Research, 2020; 20: 841. 